\title{
The modes of magnetic field generation in a low-mode model of $\alpha \Omega$-dynamo with $\alpha$-generator varying intensity regulated by a function with an alternating kernel
}

\author{
Olga V. Sheremetyeva ${ }^{1, *}$ and Anna N. Godomskaya ${ }^{2, * *}$ \\ ${ }^{1}$ Institute of Cosmophysical Research and Radio Wave Propagation FEB RAS, RU-684034, Paratunka, \\ Elizovskiy district, Kamchatka region, Russian Federation \\ ${ }^{2}$ Municipal budgetary institution of supplementary education «Center«Luch»», RU-684000, Yelizovo, \\ Elizovskiy district, Kamchatka region, Russian Federation
}

\begin{abstract}
The low-mode model $\alpha \Omega$-dynamo is used in this paper to simulate the modes of magnetic field generation with insignificant changes in the velocity field of a viscous fluid. In the framework of those model the $\alpha$-effect intensity is regulated by the process that is included in the magnetohydrodynamic system (MHD-system) as an additive correction as a functional $Z(t)$ depended on the magnetic field energy. Function that determines damped oscillations with variable damping frequency and constant damping coefficient, taken equal to one, is selected as kernel $J(t)$ of functional $Z(t)$. The research of the behavior of the magnetic field is carried out on large time scales, therefore, a rescaled and dimensionless MHD-system with the unit of time iquel the time of the magnetic field dissipation $\left(10^{4}\right.$ years $)$ for numerical calculations is used. The control parameters of the system are the Reynolds number and the amplitude of the $\alpha$-effect, that include information about the large-scale and turbulent generators, respectively. Numerical simulation of the magnetic field generation modes was carried out for the values of the damping coefficient $b=1$ and frequency $a=0.1,0.5,1,5,10$. According to the results of numerical simulation, an increase in the values of the damping frequency, when the damping coefficient is equal to one, is characterized by a decrease in the inhibitory effect of the process $Z(t)$ on the $\alpha$-effect and an increase in the region of divergence of the magnetic field on the phase plane of the control parameters. In a comparative analysis with the results of the authors' work, where the change of the $\alpha$-effect intensity was determined by the function $Z(t)$ with an exponential kernel and the same value of the damping coefficient, the following differences were noted: an increase in oscillations in both a magnetic and a velocity fields, the appearance of a chaotic regime of magnetic field generation at the value of the damping frequency equal to one, and also insignificant narrowing of the region of $\alpha$-effect suppression at values of the damping frequency increasing to one.
\end{abstract}

Keywords: $\alpha \Omega$-dynamo, magnetohydrodynamic system, $\alpha$-effect additive correction, $\alpha$-effect intensity variation, modes of magnetic field generation, reversal.

\footnotetext{
*e-mail: sheremeteva@ikir.ru

**e-mail: anna_antonenko@mail.ru
} 


\section{Introduction}

The magnetic field affects all spheres of human activity, and a change in the sign of the dipole component of the field (a change of polarity or reversal) can lead to global changes on the planet, therefore, constantly measurements of the magnetic field are being carried out and models of convection in the liquid core that can generating magnetic fields similar to real ones are being built [1-3]. Such models are used to study the nature of the occurrence of the magnetic field, its structure and to make predictions about the possible changes in the field in the near future. Despite of achievements in the theory of geomagnetism, the problem of the Earth's magnetic field origin (and other space objects) cannot be considered as completely solved, because the exact solution of the geodynamo equations is unknown. In this regard, the task of studying various approximate solutions for the generation of a geomagnetic field close in morphology to the observed one remains urgent.

The magnetic fields of space objects undergo changes over time both of a chaotic (for example, the Earth) and of a periodic nature (for example, the Sun), including those with reversals. The duration of the time intervals between reversals (polarity intervals) may differ by several orders of magnitude [4]. Model studies of the behavior of MHD-systems are limited by computational capabilities. Direct numerical modeling allows us to either solve the problem of constructing MHD-flows on small time scales and reproduce numerous inversion modes, but does not allow us to understand their causes [5, 6], or reproduce the evolution of large-scale spatial structures and explain the physical cause, signs, and most important properties of the phenomenon of polarity (reversal) [1, 7-9]. In the models of the second type, simple dynamical systems of small dimension are used, which reduces the number of variable parameters, some of which carry information about the magnetic field and allow us to understand the nature of the changes occurring in it.

The main mechanisms describing the dynamo systems of space objects are $\alpha \Omega, \alpha^{2}$ and the most common $\alpha^{2} \Omega[8,10,11]$. The model $\alpha \Omega$-dynamo is used to describe an Earth dynamo, whose characteristic properties are a strong differential rotation of the object and the turbulent nature of the conducting medium motion [7, 8, 10-14].

This work is a continuation of the cycle of long-term research on the dynamo theory conducted by the laboratory of the IKIR FEB RAS [15]. Within the framework of the low-mode model $\alpha \Omega$-dynamo $[14,16]$ with the introduced additive correction of the $\alpha$-effect intensity, the modes of magnetic field generation are simulated. The investigation is carried out on time scales comparable to the time of magnetic field attenuation, therefore, a rescaled and dimensionless MHD-system is used for the study, which reduces the possibility of computational errors during calculations over long time intervals, overloading numerical solutions with large values and shifting to «machine» infinity. The control parameters of the system are the Reynolds number and the $\alpha$-effect amplitude, which contain information about largescale and turbulent generators, respectively. The $\alpha$-effect intensity is regulated by a process that is determined by a functional depends on time and on two parameters - the coefficient $b$ and the frequency $a$ of damping.

The variation of the two control parameters responsible for the effect of each of the generators and the parameter $a$ of the process that inhibition the $\alpha$-effect intensity makes it possible to simulate various modes of magnetic field generation, including those with a change in the polarity of the magnetic field that is not associated with the rearrangement of convection. The analysis of the simulation results and their comparison with the phase portraits obtained for the exponential kernel in the paper [17] is carried out. 


\section{Formulation of the problem}

We'll give a summery description of the model, a complete investigation of which and also the main provisions and limitations are presented in the work [17]. The investigation is conducted within the framework of the model $\alpha \Omega$-dynamo. We assume that the liquid core of the Earth is bounded by a spherical shell ( $r_{1}-$ inner and $r_{2}-$ outer radii of the shell) rotating with a constant angular velocity $\boldsymbol{\Omega}$ (values varying within $10^{-1} \div 101 / \mathrm{s}$ ).

The velocity field $\mathbf{v}$ of a viscous incompressible fluid of the outer core and the generated magnetic field are axially symmetric. At the boundaries of the shell, the velocity field of a viscous liquid $\mathbf{v}$ is zero (boundary conditions of adhesion), the magnetic permeability of the inner core is equal to the permeability of the outer core, the medium beyond the boundary of the outer core is not conductive. In accordance with the accepted dynamo model, the average flow of $\overline{\mathbf{v}}$ has the character of differential rotation in the field of external forces $[7,8,11,12]$.

The physical parameters of the shell are assumed to be unchanged and the turbulence in the core is isotropic. The turbulent $\alpha$-effect is antisymmetric with respect to the equator and a scalar parametrization in the form of a function $\alpha(r, \theta)=\alpha_{0} a(r) \cos \theta=\alpha_{0} \alpha^{\prime}(r, \theta)$ is used to represent it, where the positive coefficient $\alpha_{0}$ defines a constant intensity $\alpha$-effect, $\alpha^{\prime}(r, \theta)$ - dimensionless quantity, $a(r)$ - radial component, that satisfies the following condition $r_{1} \leq|a(r)| \leq r_{2}$. Based on the accepted constraints, the low-mode model is described by MHD-equations including the Navier-Stokes equation, the magnetic field induction equation B taking into account the turbulent $\alpha$-effect, the conditions of continuity of the velocity field $\mathbf{v}$ and the solenoidality of the magnetic field, as well as boundary conditions, and in the Boussinesq approximation takes the form:

$$
\begin{aligned}
& \frac{\partial \mathbf{v}}{\partial t}+(\mathbf{v} \nabla) \mathbf{v}+\mathbf{f}_{c}=v \Delta \mathbf{v}-\frac{1}{\rho_{0}} \nabla P-\mathbf{f}_{K}+\mathbf{f}_{o u t}+\mathbf{f}_{L}, \\
& \frac{\partial \mathbf{B}}{\partial t}=\nabla \times(\mathbf{v} \times \mathbf{B})+\nabla \times(\alpha(r, \theta) \mathbf{B})+v_{m} \Delta \mathbf{B}, \\
& \nabla \mathbf{v}=0, \\
& \nabla \mathbf{B}=0, \\
& \mathbf{v}\left(\mathbf{r}_{1}\right)=\mathbf{v}\left(\mathbf{r}_{2}\right)=\mathbf{0},
\end{aligned}
$$

where $\mathbf{f}_{c}$ is centrifugal acceleration forces, $\mathbf{f}_{K}$ is mass density of the Coriolis force, $\mathbf{f}_{\text {out }}$ is mass density of the external force field (source of poloidal velocity), $\mathbf{f}_{L}$ is mass density of the Lorentz forces, $v$ is kinematic viscosity (limits of change $10^{-6} \div 10^{2} \mathrm{~m}^{2} / \mathrm{s}$ ), $\rho_{0}=7 \cdot 10^{3} \mathrm{~kg} / \mathrm{m}^{3}$ is density, $P$ is pressure, $v_{m}$ is magnetic viscosity (varies within $1 \div 20 \mathrm{~m}^{2} / \mathrm{s}$ ), $\mathbf{r}_{1}$ and $\mathbf{r}_{2}$ are radiivectors of the inner and outer boundaries of the spherical shell of the liquid core, respectively.

We introduce the characteristic values of the velocity of $u_{0}[\mathrm{~m} / \mathrm{s}]$ and the linear size of the region of $L[\mathrm{~m}]$ (the radius of the outer core $r_{2}$ ), through which we express the units of magnetic induction $B_{0}[\mathrm{Tl}]$, pressure $P_{0}[\mathrm{~Pa}]$, the mass density of external forces $f_{0}[\mathrm{~N} / \mathrm{kg}]$ and angular velocity $\Omega_{0}[1 / \mathrm{s}]$. Let is move on to a new system of units, where as a unit of time we use the time of dissipation of the magnetic field $L^{2} / v_{m}$ [s], the order of which is $10^{4}$ years, and as the control parameters we take the magnetic Reynolds number $\operatorname{Re}_{m}=\frac{u_{0} L}{v_{m}}$, responsible for the effect of a large-scale generator, and the dimensionless amplitude of the $\alpha$-effect $R_{\alpha}=\frac{\alpha_{0} L}{v_{m}}$, which contains information about the turbulent generator [17]. Then, in the rescaled form, the system (1) will be written as follows 


$$
\begin{aligned}
& \frac{\partial \mathbf{v}}{\partial t}=P_{m} \Delta \mathbf{v}-\nabla P-E^{-1} P_{m}\left(\mathbf{e}_{z} \times \mathbf{v}\right)+\mathbf{f}_{\text {out }}+(\nabla \times \mathbf{B}) \times \mathbf{B}, \\
& \left.\frac{\partial \mathbf{B}}{\partial t}=\operatorname{Re}_{m}[\nabla \times(\mathbf{v} \times \mathbf{B})]+R_{\alpha}[\nabla \times \alpha(r, \theta) \mathbf{B})\right]+\Delta \mathbf{B}, \\
& \nabla \mathbf{v}=0, \\
& \nabla \mathbf{B}=0 \\
& \mathbf{v}\left(\frac{\mathbf{r}_{1}}{L}\right)=\mathbf{v}\left(\mathbf{e}_{2}\right)=\mathbf{0} .
\end{aligned}
$$

The intensity of the $\alpha$-effect changes over time under the influence of external forces $\mathbf{f}_{\text {out }}$. The information about the $\alpha$-effect intensity in the rescaled system is contained in the control parameter $R_{\alpha}$. In our model, the process that regulates the degree of influence of the turbulent effect is defined as a functional $Z(t)$, the effect of which is determined by the quadratic form $Q(\mathbf{B}(\tau), \mathbf{B}(\tau))$, and the nature of the those effect is determined by the dimensionless kernel $J(t-\tau)$ :

$$
Z(t)=\int_{0}^{t} J(t-\tau) Q(\mathbf{B}(\tau), \mathbf{B}(\tau)) d \tau .
$$

Considering the additive correction $Z(t)$ of the $\alpha$-effect intensity the MHD-system can be written in the following form

$$
\begin{aligned}
& \frac{\partial \mathbf{v}}{\partial t}=P_{m} \Delta \mathbf{v}-\nabla P-E^{-1} P_{m}\left(\mathbf{e}_{z} \times \mathbf{v}\right)+\mathbf{f}_{\text {out }}+(\nabla \times \mathbf{B}) \times \mathbf{B}, \\
& \left.\frac{\partial \mathbf{B}}{\partial t}=\operatorname{Re}_{m}[\nabla \times(\mathbf{v} \times \mathbf{B})]+\left(R_{\alpha}-Z(t)\right)[\nabla \times \alpha(r, \theta) \mathbf{B})\right]+\Delta \mathbf{B}, \\
& \nabla \mathbf{v}=0 \\
& \nabla \mathbf{B}=0 \\
& \mathbf{v}\left(\frac{\mathbf{r}_{1}}{L}\right)=\mathbf{v}\left(\mathbf{e}_{2}\right)=\mathbf{0} .
\end{aligned}
$$

\section{The numerical simulation}

One of the approaches to the investigation of MHD-systems is the construction of low-mode approximations of the Galerkin type [1]. Note, that different versions of the dynamo mechanism have a unified approach in that the source of the toroidal (poloidal) component of the field $\mathbf{B}$ is the nonlinear interaction of the poloidal (toroidal) component of the same field with the fluid flow $[8,11]$. In this regard, one of the possible truncations is the selection of four components (modes): one hydrodynamic and three magnetic - one toroidal and two poloidal $[14,18]$, which we use for the numerical solution of the system (4),

$$
\begin{aligned}
\mathbf{v}=u(t) \mathbf{v}_{\mathbf{0}} & =u(t)\left(\alpha_{1} \mathbf{v}_{0,1,0}^{T}+\alpha_{2} \mathbf{v}_{0,2,0}^{P}+\alpha_{3} \mathbf{v}_{0,3,0}^{T}+\alpha_{11} \mathbf{v}_{1,1,0}^{T}+\alpha_{13} \mathbf{v}_{1,3,0}^{T}\right), \\
\mathbf{B} & =B_{2}^{T}(t) \mathbf{B}_{0,2,0}^{T}(\mathbf{r})+B_{1}^{P}(t) \mathbf{B}_{0,1,0}^{P}(\mathbf{r})+B_{3}^{P}(t) \mathbf{B}_{0,3,0}^{P}(\mathbf{r}),
\end{aligned}
$$

where $\mathbf{v}_{\mathbf{0}}$ is the Poincare mode [19], $u(t)$ is the velocity amplitude, $\mathbf{B}_{0,1,0}^{P}(\mathbf{r})$ is the dipole component of the magnetic field, which under the influence of differential rotation generates toroidal $\mathbf{B}_{0,2,0}^{T}(\mathbf{r})$ and poloidal $\mathbf{B}_{0,3,0}^{P}(\mathbf{r})$ components. We consider the components of the velocity and of the magnetic fields to be independent.

The functional $Z(t)(3)$ is given as the integral of the product of the magnetic field energy and the alternating function $J(t)$, which determines the damped oscillations with a damping 
coefficient $b$ and the frequency of damped oscillations $a$,

$$
Z(t)=\int_{0}^{t} e^{-b(t-\tau)} \cos (a(t-\tau)) \mathbf{B}^{2}(\tau) d \tau .
$$

The choice of an alternating damping function as the kernel $J(t)$ defines a process with a finite waiting time (memory), the overwhelming influence of which on the change in the intensity of the $\alpha$-effect is included at the initial moment of time and gradually accumulates during the waiting time $t_{0}$. Variation of the parameters changes the influence nature of the inhibition functional. In this paper, we will investigate the model with a constant damping coefficient of $b$ and a variation of the parameter $a$, which is responsible for the frequency of changing the sign of the functional kernel. The waiting time $t_{0}$ was calculated based on the condition that $95 \%$ of the inhibition functional is concentrated on the segment $\left[0, t_{0}\right]$,

$$
\int_{0}^{t_{0}} e^{-b t}|\cos (a t)| d t=0.95 \int_{0}^{\infty} e^{-b t}|\cos (a t)| d t .
$$

We substitute the decompositions of (5) and the function (6) into the system (4), after which we apply the Galerkin method. As a result of the transformations, we obtain a system that is then used for numerical implementation by the backward Euler method,

$$
\begin{aligned}
& \frac{\partial u}{\partial t}=-P_{m} u(t) \sum_{k} \alpha_{k}^{2} \lambda_{k}+f_{\text {out }}+\sum_{i, j, k} \alpha_{i} L_{i j k} B_{j} B_{k}, \\
& \frac{\partial B_{i}}{\partial t}=R e_{m} u(t) \sum_{j, k} \alpha_{j} W_{i j k} B_{k}-\mu_{i} B_{i}+\left(R_{\alpha}-Z\right) \sum_{k} W_{i k}^{\alpha} B_{k}, \\
& \frac{\partial Z}{\partial t}=\sum_{k} B_{k}^{2}-b Z-a Z_{s}, \frac{\partial Z_{s}}{\partial t}=a Z-b Z_{s}, i, j, k=1,2,3 .
\end{aligned}
$$

where $\lambda_{i}$ is eigenvalues of the Poincare mode [20], coefficients $L_{i j k}, W_{i j k}, W_{i j}^{\alpha}$ are the volume integrals of the fields under consideration [21], $\mu_{i}$ is the viscous dissipation parameter [20], the values of the coefficients $\alpha_{i}$ were calculated from the Poincare problem on free small oscillation of a viscous rotating fluid [19].

The accepted value of the sampling step $h$ is two orders of magnitude less than the smallest of the damping times of the magnetic field and the velocity field, which are determined by the values of the coefficients $\lambda_{i}$ and $\mu_{i}$. The choice of initial conditions ensures the output of the system (8) from the rest point at the initial moment of time $t=0$ and further mutual generation of the components of the magnetic and the velocity fields

$$
u(0)=1, B_{2}^{T}(0)=0, B_{1}^{P}(0)=1, B_{3}^{P}(0)=0, Z(0)=0, Z_{s}(0)=0 .
$$

Table 1. The parameters depending on the function $\mathrm{J}(\mathrm{t})$

\begin{tabular}{|c||c|c|c|}
\hline$a$ & $t_{0}$ & $\int_{0}^{\infty}|J(t)| d t$ & $\int_{0}^{\infty} J(t) d t$ \\
\hline 0.1 & 2.922 & 0.99 & 0.99 \\
0.5 & 1.871 & 0.817 & 0.8 \\
\hline 1 & 1.108 & 0.604 & 0.5 \\
\hline
\end{tabular}


The damping effect in the velocity field is compensated by the influence of external forces, therefore, in the numerical implementation, the value of the mass density of external forces $f_{\text {out }}$ is assumed to be equal to the coefficient at $u(t)$ [17]. The numerical model is considered for control parameters, that varying in the following ranges $\operatorname{Re}_{m} \in(0,1000]$ and $R_{\alpha} \in(0,100]$, in increments of five. Damping frequency values $a$ and the characteristics of the inhibition process $Z(t)$, depened on it, are presented in table 1 , the damping coefficient $b$ is constant and assumed to be equal to one. The results of numerical simulation for the given model parameters are presented on the phase plane of the control parameters $R e_{m}, R_{\alpha}$ (fig. 2).

Figure 1. The nature of magnetic field generation depending on the parameters $R_{\alpha}$ and $R e_{m}$. The $\alpha$-effect intensity constant $-\alpha_{0}$. The white region is the generation of the increases infinitely magnetic field, the red one - is the generation of a damped magnetic field, the green is the steady mode of magnetic field generation [17].

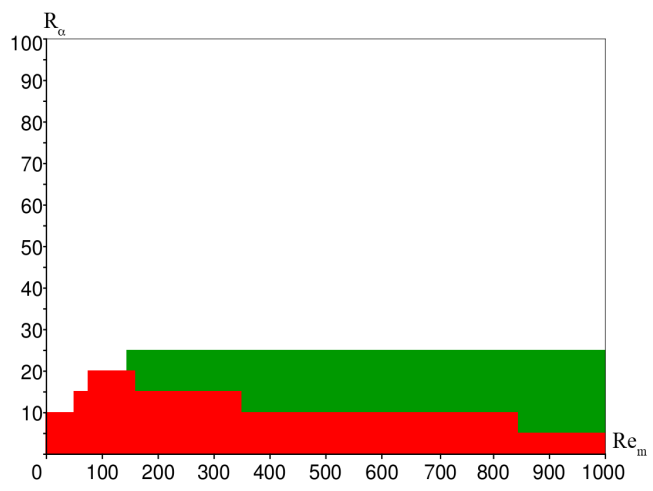

\section{Simulation results and discussion}

The simulation results showed that the inclusion in the MHD-system of influence that regulates the $\alpha$-effect intensity in the form of a process determined by the functional $Z(t)$ with an alternating kernel, lead to a narrowing of the divergence region of the magnetic field and to an increase in the regions with modes of magnetic field generation without unlimited growth, including those with reversals, under the condition of a weakly varying velocity field. And also it's caused the emergence of new modes of magnetic field generation, in addition to damped, steady and unlimited growth of the magnetic field, which are formed in the case of a constant intensity of the $\alpha$-effect, i. e. in the absence of a supression effect (fig. 1) [17].

We compare the simulation results (fig. 2 b, c, d) with those obtained in the case of a constant intensity of the $\alpha$-effect. With a constant damping coefficient $b=1$ and an increase in the damping frequency $a$ of the alternating kernel $J(t)$ of the functional $Z(t)$ on the phase plane of the control parameters $R e_{m}, R_{\alpha}$ the region of the damped generation mode (with or without oscillations) is preserved within the same boundaries, a slight increase in which occurs, when $a=0.1$ and $R e_{m} \in[100,250], R_{\alpha} \in[15,20]$, as a result of inhibition of the steady mode of magnetic field generation. For the values of the damping frequency $a \leq 1$, the divergence region of the magnetic field decreases becouse the regions of steady, steady-state, chaotic, vacillation and dynamo-burst modes appearance on the interval $R_{\alpha} \in[10,80]$. The largest part of the phase plane (in the $R e_{m} \in[80,1000]$ interval) is occupied by the region of the steady mode of magnetic field generation. At the values of $R e_{m}<100$, small regions of the steady-state and vacillation modes are formed on the phase plane, and for $a=1$ are formed a dynamo-burst regions. If the values of $R e_{m}>100$ and $a=1$, then a small region of chaotic mode generation is formed. With a further increase in the damping frequency $a$ to a value of 10, the divergence region of the magnetic field increases again due to a significant decrease in the region of the steady mode, although in this case the regions of the steadystate, chaotic and vacillation modes are increase. Note, that at values of $a \in(1 ; 10]$, changes 

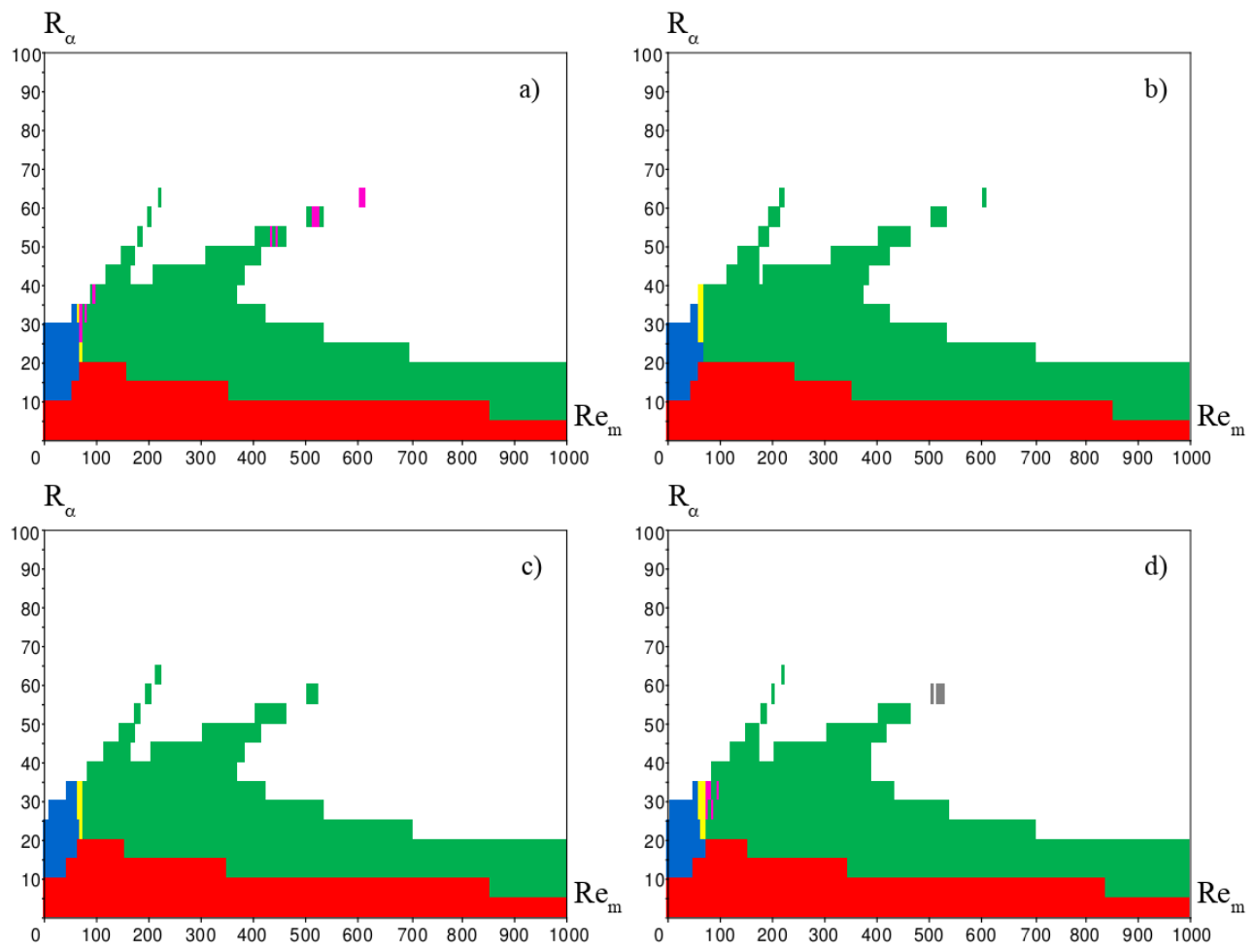

Figure 2. The nature of magnetic field generation depending on the parameters $R_{\alpha}$ and $R e_{m}$. The $\alpha$ effect intensity is defined by the function $Z(t)$ with the kernel: a) $J(t)=e^{-t}[17]$, b) $J(t)=e^{-t} \cos (0.1 t)$, c) $J(t)=e^{-t} \cos (0.5 t)$, d) $J(t)=e^{-t} \cos (t)$. The white region is the generation of the increases infinitely magnetic field, the red one - is the generation of a damped magnetic field, the green is the steady mode of magnetic field generation, the blue is the steady-state mode, the yellow is the vacillation, the lilac is the dynamo-burst, the grey is the chaotic magnetic field generation mode.

of the magnetic field components amplitudes occur with a high frequency of the oscillation, which causes significant changes in the amplitude of the velocity field $(0.25 \leq \Delta u \leq 1)$ up to a sign reversal for $a=10$. Thus, the applicability of the consideration model is constraints by the values of the parameter $a \leq 1$, therefore, the further discussion and comparison of the simulation results will be carried out for these values of the damping frequency.

Comparison of the simulation results for the values of the parameter $a$ of the kernel $J(t)$ of the inhibition functional $Z(t)$ vary from 0.1 to 1 , revealed a tendency of decrease of the regions of limited oscillations and of the insignificant change their boundaries (fig. 2 b, c, d). For all of the parameter $a$ values the steady-state mode on the interval $R e_{m} \in(0,100)$ is replaced by the vacillation mode, which forms a small transition region, and then - by the steady mode, which forms the main region of the limited oscillations within the values of the control parameter $R e_{m} \in[100,1000]$. At the value of the damping frequency $a=1$ in the transition region (the region of the vacillation mode), the small inclusions of the dynamo-burst regions appear in front of the region of the steady mode of magnetic field generation and the region of the steady mode, located on the phase plane in the square $R e_{m} \in[510,530], R_{\alpha} \in[55,60]$ at $a<1$, is replaced by the region of the chaotic mode of magnetic field generation. 
Changes on the phase plane are related to the characteristics of the inhibition functional $Z(t)(6)$, depended on the choice of the kernel $J(t)$ and the values of its parameters (table 1). An increase in the values of the damping frequency $a$ at a constant damping coefficient $b=1$ leads to a decrease in the values of the waiting time $t_{0}$ and integrals of the function $J(t)$ and its modulus. However, in the case of $a=0.1$, the values of the integrals practically coincide (up to the eighth decimal place), that indicates a very weak effect of damped oscillations in the tail on the total value of the integral. In other cases, this influence is more significant and this does not allow suppressing the $\alpha$-effect in a larger region than at $a=0.1$, which is displayed on the narrowing of the region of magnetic field generation limited modes in the interval $R_{\alpha} \in[50,70]$ on the phase plane. A more significant influence of the tail for $a=1$ also causes the appearance of new modes of generation: dynamo-burst and chaotic. Thus, the short-term intense integral action of the process $Z(t)$ during the waiting time $\left(t_{0} \approx 3\right)$, which changes insignificantly during the rest of the time, leads to suppression of the field divergence in the most of the considered region on the phase plane control parameters. Nevertheless, in all cases, the main contribution to the suppression of the $\alpha$-effect is made at the initial timing steps, where the function $J(t)$ is positive and the integral of it takes the greatest value, which in a large extent affects the similarity of the patterns on phase plane for all considered values of the damping frequency $a$.

If we compare the obtained results with the simulation one's for the inhibition functional $Z(t)$ with an exponential kernel and damping coefficient $b=1$ (fig. 2 a) [17], then the obtained patterns on the phase plane are similar. On the whole, the contours of the main regions (steady, damping, steady-state modes) are preserved. But this patterns have also differences. For values $a=0.1-0.5$ (fig. $2 \mathrm{~b}, \mathrm{c}$ ), there are no dynamo-burst regions. Instead of dynamo-burst regions, in one case, there is a vacillation region in the transition from steadystate regime region to steady one, in the other, there is a the region of a steady regime in the transition to the region of divergence of the magnetic field. For the case $a=1$ in the transition to the divergence region of the field, the dynamo-burst is replaced by the chaotic regime region (fig. $2 \mathrm{~d}$ ). Thus, the use of an oscillating damping function instead of an exponential one as the kernel $J(t)$ of the functional $Z(t)$ caused to an increase in oscillations in both the magnetic field and the velocity field, that, on the one side, reduces or exclud the possibility of appearance of a dynamo-burst regions, on the other side, leads to irregular behavior and to the emergence of a chaotic regime at the boundary with the divergence region of the magnetic field.

\section{Conclusion}

The consideration of the MHD-system with an additive correction of the $\alpha$-effect intensity in the form of a functional $Z(t)$ with an alternating kernel $J(t)$ induced an increase in the magnetic field oscillations frequency, which led to an increase in the amplitude and/or the frequency of oscillations in the velocity field, including those with a change in sign at values of the damping frequency $1<a \leq 10$. Thus, in this case the model on consideration is applicable only at values of the damping frequency $a \leq 1$.

Using the process $Z(t)$ for regulate $\alpha$-effect intensity led to a decrease in the magnetic field divergence region compared with the case of constant intensity of $\alpha$-effect and to emergence of new modes of magnetic field generation: vacillation, dynamo-burst, chaotic, steady-state.

The nature of the influence of the process $Z(t)$ on the $\alpha$-effect intensity is determined by the tow parameters of the kernel $J(t)$ - the damping coefficient and frequency. The waiting time $t_{0}$ decreases with increasing frequency values $a$, when the damping coefficient is constant $b=1$. As a consequence, a greater contribution is made by alternating tails, the influence on the $\alpha$-effect intensity decreases, which leads to an increase in the magnetic field 
divergence region, to change in the configuration of the regions of the obtained modes of magnetic field generation and to emergence of new modes of magnetic field generation under conditions of a weakly varying velosity field.

The characteristics of the alternating kernel of functional $Z(t)$ at the permissible values of the damping frequency differ insignificantly from the those of the exponential kernel. Therefore, the patterns on the phase planes in both cases are similar in the configuration and in the regions location of the obtained modes of magnetic field generation. However, in our case, a decrease in the damping frequency $a$ leads to the disappearance of the dynamo-burst regions obtained due to choosing the exponential kernel and to an increase in the vacillation region, while an increase in frequency leads to a decrease in the vacillation region and to the appearance of the chaotic and dynamo-burst regions.

The paper was carried out within the framework on the subject «Physical processes in the system of near space and geospheres under solar and lithospheric influences» (AAAA-A21121011290003-0).

\section{References}

[1] E. B. Gledzer, F. V. Dolzhanskiy, A. M. Obukhov, Sistemy gidrodinamicheskogo tipa i ikh primenenie [Hydrodynamic Type Systems and Their Application] (Nauka, Moscow, 1981) 368 [in Russian]

[2] M. Kono, P.H. Roberts, Reviews of Geophysics 40, B1-B41 (2002)

[3] D. D. Sokoloff, R. A. Stepanov, P. G. Frick, UFN 184, 313-335 (2014) https://doi.org/10.3367/UFNr.0184.201403g.0313

[4] R. T. Merril, M. W. McElhinny, P. L. McFadden, The Magnetic Field of the Earth: Paleomagnetism, the Core, and the Deep Mantle (Academic Press, London, 1996) 531

[5] G. Glatzmaier, P. Roberts, Nature 377, 203-209 (1995)

[6] W. Kuang, J. Bloxman, Nature 389, 371-374 (1997)

[7] M. Steenbek, F. Krause, Astron. Nachr. 291, 49-84 (1969)

[8] Ya. B. Zeldovich, A. A.Rusmaikin, D.D. Sokoloff, Magnetic fields in astrophysics. The Fluid Mechanics of Astrophysics and Geophysics (Gordon and Breach, New York, 1983) 382

[9] L. K. Feschenko, G. M. Vodinchar, Nonlinear Processes in Geophysics 22, 361-369 (2015) https://doi.org/10.5194/npg-22-361-2015

[10] E.N. Parker, Astrophys. J. 122, 293-314 (1955)

[11] F. Krause, K.H. Rädler, Mean-filed magnetohydrodynamics and dynamo theory (Pergamon Press, Oxford, 1980) 271

[12] M. Steenbek, F. Krause, K.H. Rädler, Z. Naturforsch. 21, 369-376 (1996)

[13] D. D. Sokoloff, S. N. Nefedov, Num. Meth. Prog. 8, 195-204 (2007)

[14] G. M. Vodinchar, L. K. Feshchenko, Magnetohydrodynamics 52, 287-300 (2016)

[15] G. M. Vodinchar, R. I. Parovik, A. S. Perezhogin, O. V. Sheremetyeva, Istoriya nauki i tehniki [History of Science and Engineering] 8, 100-112 (2017) [in Russian]

[16] G. M. Vodinchar, Vestnik KRAUNTS. Fiz.-Mat. Nauki [Bulletin KRASEC. Phys. \& Math. Sci.] 2(7), 33-42 (2017) [in Russian] https://doi.org/10.18454/2079-6641-2013-72-33-42

[17] O. V. Sheremetyeva, A. N. Godomskaya, Bulletin of the South Ural State University, Series «Mathematical Modelling, Programming \& Computer Software» 14, 27-38 (2021) https://doi.org/10.14529/mmp210203 
[18] G. M. Vodinchar, A. N. Godomskaya, O. V. Sheremetyeva, E3S Web of Conferences, 11, 00024 (2016) https://doi.org/10.1051/e3sconf/ 20161100024

[19] G. M. Vodinchar, L. K. Feshenko, Bulletin KRASEC. Phys. \& Math. Sci., 2(11), 41-50 (2015) https://doi.org/10.18454/2313-0156-2015-11-2-41-50

[20] G. M. Vodinchar, Baza dannyh «Parametry sobstvennyh mod svobodnyh kolebanij MGD polej v yadre Zemli» [Database «Parameters of natural modes of normal oscillations of MHD fields in the Earth's core»], RU 2019620054 (2019) [in Russian]

[21] G. M. Vodinchar, L. K. Feshenko, Biblioteka programm dlya issledovaniya «Malomodovoj modeli geodinamo» [Library of programs for researching «Low-mode geodynamo model» «LowModedGeodinamoModel»], RU 50201100092 (2011) [in Russian] 